EL MAESTRO UECHI KANEI DIRIGIENDO LA PRÁCTICA DE

SANCHIN DE RYMARUK. DOJO DE FUTENMA, 1982

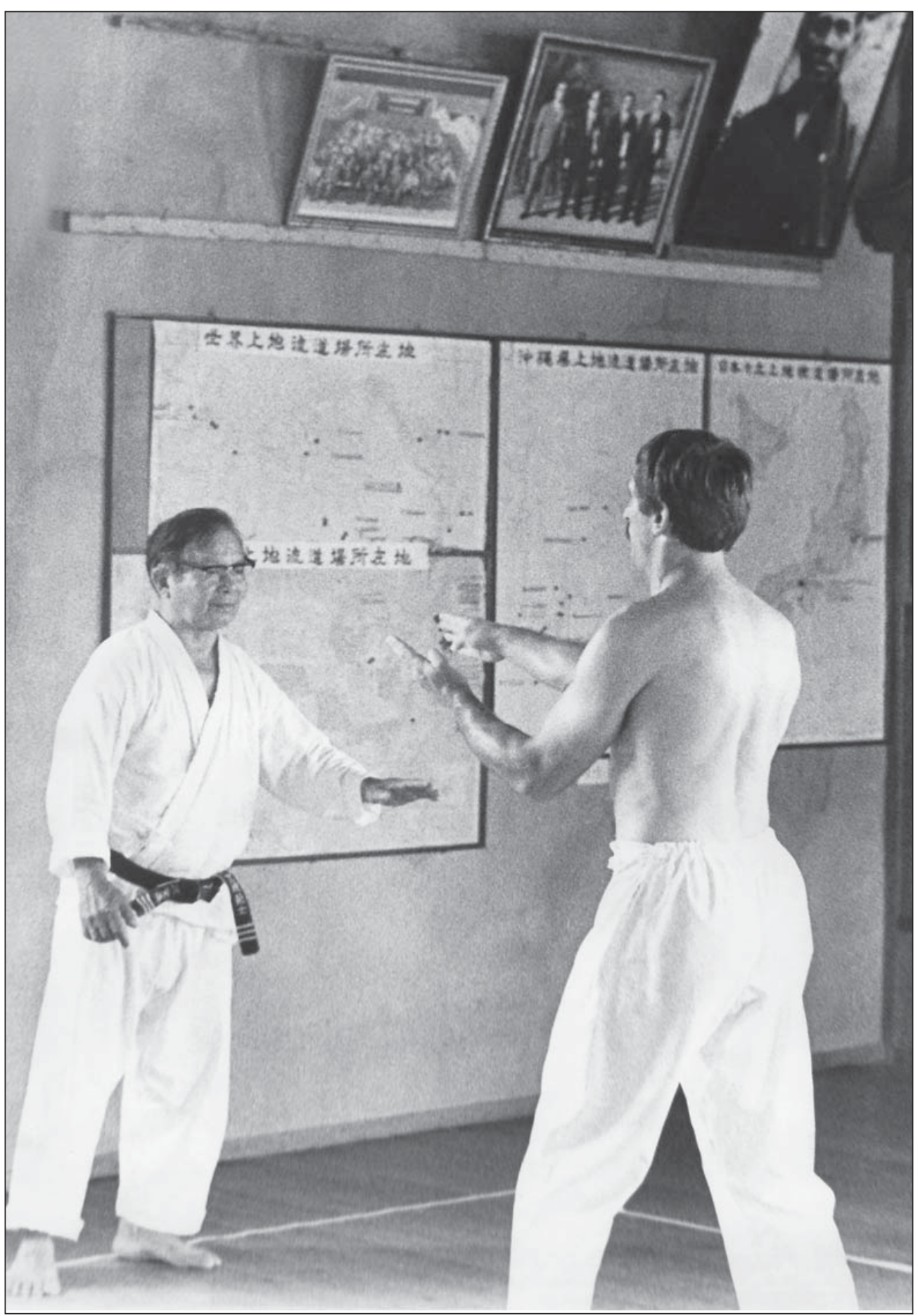

\section{Resumen}

Este artículo presenta "Defendiendo en las Cuatro Direcciones", técnicas que añaden un desafío al Uechi-ryu, aunque se hayan incorporado y construido sobre las katas tradicionales que forman los pilares del estilo. Realizar cambios dentro de los métodos de entrenamiento de un estilo simplemente para ser diferente sin ningún otro propósito es de poco valor y realmente destructivo para el sistema. Este artículo describe una aportación realista y llena de significado a la práctica del hojoundo, los ejercicios formales que incorporan movimientos básicos. "Defendiendo en las Cuatro Direcciones" está pensado para estudiantes avanzados que han dominado los fundamentos del Uechi-ryu. Además, la práctica encaja bien para su adaptación y encaje en otros estilos de artes marciales. 


\title{
LAS CUATRO DIRECCIONES: DESARROLLANDO LOS EJERCICIOS DE HOJOUNDO DEL UECHI-RYU PARA ESTUDIANTES AVANZADOS
}

\author{
IHOR RYMARUK
}

$\Delta \boldsymbol{\nabla} \mathbf{\Delta}$

\section{Breves antecedentes del Uechi-ryu}

El Uechi-ryu es uno de los cuatro principales estilos de kárate de la isla de Okinawa. Es singularmente diferente en que no ha perdido su semejanza con sus raíces del sur de China. En la esencia del estilo están las tres katas que Uechi Kanbun (1877-1948), el fundador del Uechi-ryu, trajo de China después de pasar 13 años en el continente como estudiante, y que luego enseñó en su propia escuela -sanchin, sesan, y sanseru-. En 1940, los estudiantes de Kanbun renombraron la escuela de Uechi de Pangainoon-ryu Karatejutsu Kenkyu-jo (Lugar de Estudio de las Técnicas de Mano Vacía del Estilo Mitad-Duro, Mitad-Blando) a Uechi-ryu Karatejutsu (el Arte de Uechi de la Mano Vacía).

Uechi Kanei (1911-1991), el hijo mayor de Kanbun, dio permiso a un grupo de practicantes veteranos para reunir las técnicas de combate que enseñaba Kanbun. Entre 1950 y 1960, el Uechi-ryu alcanzó, en su mayor parte, su formato actual. Se desarrollaron cinco katas iniciales e intermedias basadas en las tres katas originales. Se añadieron algunos ejercicios de calentamiento, y se extrapolaron las técnicas singulares del estilo de combate de Kanbun, con su sello particular, para constituir los ejercicios formales (hojoundo). La esencia de las katas y técnicas del Uechi-ryu ha sido sólida durante más de cincuenta años. Durante estos años se desarrollaron varios ejercicios entre dos personas, pero no hubo cambios globales en el modo en que los estudiantes practicaban los ejercicios esenciales. La práctica del Uechi-ryu en las principales escuelas de Okinawa es constante, aunque ninguna de ellas es una copia exacta de las otras.

Entrenar en Okinawa es un sueño para la mayoría de entusiastas del kárate; sin embargo, las dificultades para viajar, el idioma, y el tiempo impiden que la mayoría realice ese sueño. Sólo unos pocos individuos fervorosos tuvieron el privilegio de formar parte del dojo de Uechi Kanei. Mi primer instructor cualificado de Uechi-ryu, Frank Gorman, y ahora mi actual instructor, James Thompson, enseñaron y practicaron Uechi-ryu como lo enseñó Uechi Kanei. Thompson entrenó exclusivamente en el dojo principal en la Base Aérea de Futenma bajo la tutela directa de Uechi Kanei durante casi diez años, un logro extraordinario para una persona de fuera de Okinawa en aquella época. Ser parte del dojo de Uechi Kanei fue una experiencia especial para mí junto con la conciencia de ser parte de una organización más grande que cualquier escuela. Hoy en día existen muchos desplazados de otros países que se han enamorado de la cultura de la isla y de su gente, y que practican las artes marciales de forma regular. Algunos han llegado a ser los maestros principales de sus propios dojo de Uechi-ryu.

Todas las fotografías son cortesía de Ihor Rymaruk. 


\section{Enriqueciendo la forma estándar de los ejercicios formales}

La práctica hojoundo consiste básicamente de trece combinaciones de brazo y pierna que siempre comienzan con un bloqueo, y se realiza normalmente de forma grupal sin tener en cuenta el grado. Tradicionalmente, los ejercicios de hojoundo se practican mientras se está en el mismo sitio, y los ejercicios son iguales para estudiantes novatos, intermedios y avanzados. Esto es, en el estilo Uechi-ryu no hay una progresión de dificultad para desafiar a los estudiantes más avanzados. Después de ejecutarse miles de repeticiones, la destreza entrenada se integra en la memoria muscular y se convierte en un reflejo natural o hábito. La práctica del hojoundo exige un gran esfuerzo a cualquier nivel, y es una buena sensación el ser capaz de ejecutar técnicas de forma automática sin errores. Creemos que el entrenamiento en artes marciales se compone de mente, cuerpo, y espíritu. Actualmente, nuestro destino en el viaje de nuestro entrenamiento es reaccionar con una "mente vacía". En esencia, una mente vacía no está necesariamente carente de conocimiento, pero está condicionada a responder a cualquier desafío sin pensarlo.

Mientras avancé en mi comprensión del Uechi-ryu, comencé a incorporar varias secuencias de avance en mi práctica de los ejercicios formales. La culminación de la cual es la evolución de las formas antiguas realizadas con un nuevo giro, el cual llamo "Defendiendo en las Cuatro Direcciones". La enseñanza y desarrollo de los fundamentos con los nuevos estudiantes todavía se hace de la manera tradicional, que está avalada por la misma tradición. Sin embargo, a medida que los estudiantes progresan en grado y entendimiento, parecía lógico desafiar la "zona de comodidad" del estudiante mientras atraviesan los niveles de principiante, intermedio, y avanzado. Después de todo, yo también, que soy aún un principiante, necesitaba esforzarme, explorar, y alcanzar mi propio entendimiento a diferentes niveles. Sacando a los estudiantes de su zona de comodidad al poner el hojoundo en movimiento, se sigue construyendo sobre lo que los estudiantes ya pueden realizar bien de forma estática, y ayuda a los estudiantes a descubrir, a través de este desafío, que existe una necesidad continua de aumentar los retos tanto en el plano mental como físico. La suma de ejercicios para entrenar las destrezas tiene el beneficio de mantener despierta la sed del estudiante de nueva información, y demuestra que el nivel de entrenamiento de uno mismo sólo encuentra barreras en las propias limitaciones. La evolución es una amiga, estimulada e influenciada gracias al tiempo y a la constancia del entrenamiento.

Es importante para todos los nuevos estudiantes el desarrollar y entender la forma original de los ejercicios formales como los desarrolló y practicó Uechi Kanei. Sin esta forma original, no hay estilo de Uechi-ryu. Una vez que el estudiante puede realizar los fundamentos formales fácilmente y sin ninguna duda, él o ella ya está preparado para añadir vida al régimen de entrenamiento de los ejercicios formales estáticos. Una vez que los ejercicios formales se han aprendido, se suele enseñar a los estudiantes las destrezas de combate con control de dos personas. Estas habilidades (kyu kumite) son los verdaderos comienzos para desarrollar la sincronización y la distancia, y los bloqueos y golpeos simples. Sin embargo, estas habilidades no incorporan los ejercicios formales de una forma defensiva y ofensiva práctica, y de hecho los relegan a un calentamiento para las katas, sin mucha exploración o práctica de sus verdaderos beneficios para la defensa personal real. Además, los ejercicios de hojoundo implican que todos los ataques vienen del frente. La aplicación del hojoundo debe ser entendida por los maestros, pero no debería llevar años a los estudiantes el dar el salto al nivel de su aplicación práctica.

Comencé a buscar maneras de ayudar a los estudiantes a comprender y tender puentes hacia la aplicación del hojoundo. El hojoundo se realiza en la postura del reloj de arena (sanchin) con un determinado pie adelantado. La primera modificación que integré para los principiantes de nivel intermedio fue el alternar los movimientos de brazos. Esto es, practicar un ejercicio que está pensado para ser realizado con un bloqueo del brazo izquierdo, por ejemplo, alternando izquierda y luego derecha con cada repetición. Esto modifica la fluidez de la combinación original, pero no la combinación en sí misma. Si la manera normal es hacer una cierta técnica sobre la pierna adelantada o sobre la pierna atrasada, practico la combinación del tren superior sin tener en cuenta qué pier- 
na está delante. Mantendría mi postura estática, pero alternaría las combinaciones de manos. Después de todo, los estudiantes deberían ser capaces de aplicar combinaciones que han aprendido sin atender al pie que está adelantado. Además, el beneficio añadido de alternar los lados es que tu vestimenta ya no se enrollará más en tus hombros o brazos por hacer el mismo movimiento repetitivo.

Cuando ya estuve cómodo practicando de esta manera ambidiestra estática, encontré que el realismo de los movimientos de combate aún estaba perdido, y que la forma tradicional de bloquear un pie específico no se estaba aplicando. Rara vez el combate realista es una actividad estática sin juego de pies, y es importante mantener los fundamentos del estilo. Por lo tanto, para mis estudiantes intermedios y avanzados, di un paso más y coloqué el hojoundo en movimiento, con un solo paso. Esto se consiguió avanzado hacia delante y ejecutando una combinación dada hasta que no quedaba más sala para avanzar, momento en el que avanzábamos hacia atrás ejecutando la misma combinación antes de pasar a la siguiente. Esto permitió alternar los brazos durante la práctica de la combinación sin comprometer la forma normal del hojoundo. Mientras que avanzar hacia delante y hacia atrás no es la única forma de practicar los fundamentos -como hacen muchos otros estilos durante el trabajo de las destrezas básicas- no es así precisamente como se practicaba el hojoundo tradicional de Uechi-ryu. Cuando había muchos estudiantes en clase, y el espacio estaba limitado, tenía a los estudiantes dando un paso hacia delante, ejecutar la combinación, dar un paso hacia atrás, y realizar la combinación de nuevo, de seis a diez repeticiones (A1-3). Este método funcionó bien, y el paso hacia delante daba la sensación de estar a la ofensiva, mientras que el paso hacia atrás daba la sensación de estar a la defensiva. Así, el hojoundo se asemejaba a los movimientos de una ola del océano, moviéndose hacia delante, recortando terreno/ataque, y moviéndose hacia atrás, perdiendo terreno/defensa.

El mantenimiento de una buena forma del ejercicio será probablemente un desafío para los estudiantes intermedios. Sin embargo, cuanto más competente se hace el estudiante, más fácil será para él/ella mantener su enraizamiento sanchin. A través del movimiento de avance, los estudiantes pueden visualizar más fácilmente la aplicación de cada ejercicio de hojoundo, incluso aunque todavía acepten que el ataque que están defendiendo viene desde el frente.

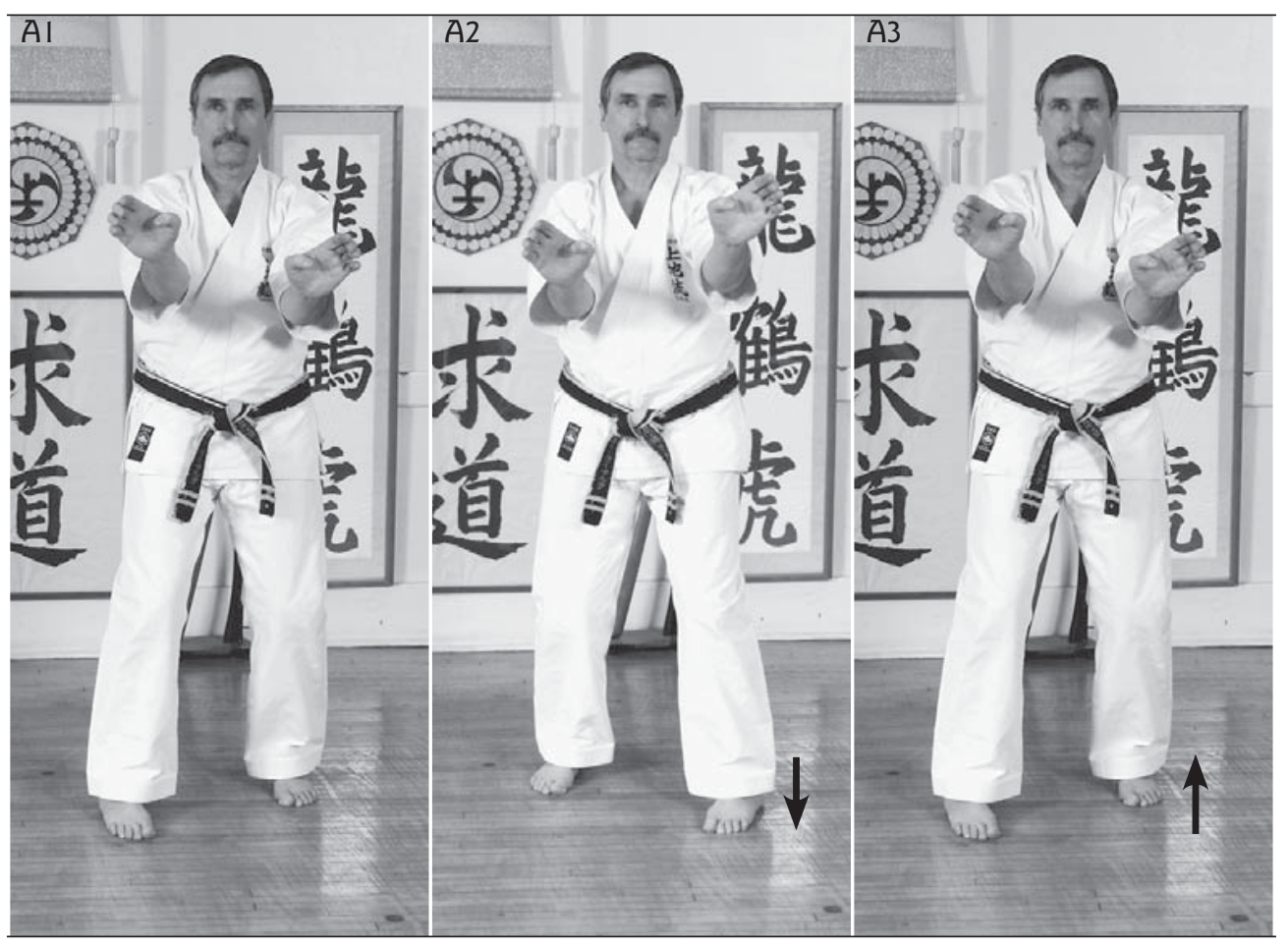

Revista de Artes Marciales Asiáticas $\diamond$ Volumen 3 Número 1 (80-91) - 2008

\section{Secuencia de los ejercicios de Hojoundo utilizados en Defendiendo en las Cuatro Direcciones}

I) bloqueo circular, patada frontal

2) bloqueo circular, puñetazo en gancho

3) puñetazo de bloqueo alto, puñetazo de bloqueo medio

4) puñetazo de bloqueo circular, puñetazo de bloqueo con la palma de la mano

5) bloqueo circular cortante, puñetazo con el revés del puño

6) bloqueo circular, codazos

7) golpeo con los dedos

8) cuatro formas de bloqueo con la muñeca

9) bloqueos en forma de colas de peces

El trabajo de pies fundamental es el avance hacia delante $y$ hacia atrás. 


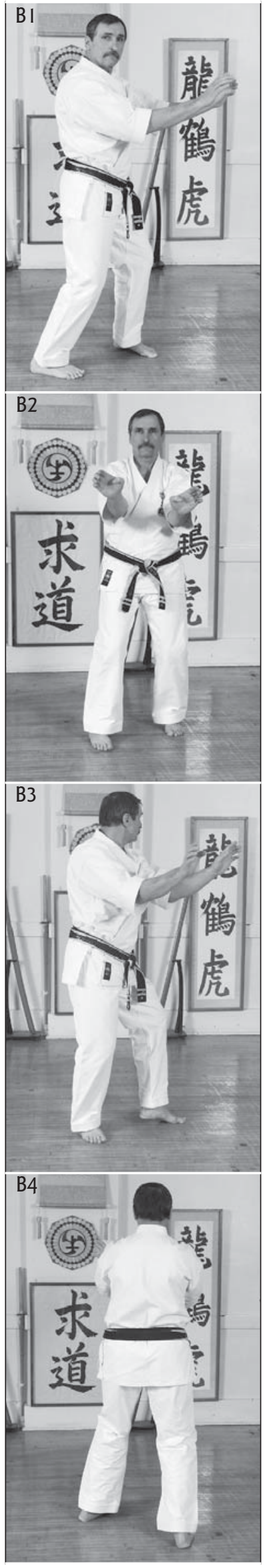

\section{Sección Técnica}

\section{Defendiendo en las Cuatro Direcciones}

La siguiente progresión lógica para la práctica del hojoundo es apreciar que los ataques pueden venir de cualquier dirección. Creé "Defendiendo en las Cuatro Direcciones" hace aproximadamente dos años cuando tutelaba y criticaba las habilidades de liderazgo y enseñanza de los estudiantes, a la vez que seguía mi propio desarrollo personal y estudiaba en la última fila al lado de los nuevos cinturones blancos. Esta técnica fue desarrollada mientras experimentaba con maneras de dar a mis ejercicios formales de Uechi-ryu un giro más práctico y real.

Este ejercicio, junto con la utilización de una fuerte visualización, aumenta la practicidad del Uechi-ryu en las situaciones de defensa personal y desafiaba incluso a los estudiantes más avanzados. Habrás entrenado para defenderte de múltiples amenazas que vengan de cualquier dirección, mientras permaneces fiel a los fundadores y fundamentos del Uechi-ryu. "Defendiendo en las Cuatro Direcciones" no es una habilidad muy complicada; simplemente no es para principiantes. Esta adición multidimensional al hojoundo convierte al Uechi-ryu en un sistema de combate cuerpo a cuerpo más real.

Para realizar el juego de pies de las "Cuatro Direcciones" necesitarás entender dos giros básicos, un giro pivote de $90^{\circ}$, y un giro de $180^{\circ}$ conocido como giro Sanchin por ejecutarse desde la posición Sanchin. Para realizar el giro pivote de $90^{\circ}$ comienza en la postura sanchin con el pie izquierdo adelantado. El dedo gordo de tu pie atrasado debería estar en línea con el talón de tu pie adelantado, y tus pies deberían estar separados aproximadamente la anchura de tus hombros. Pivota sobre la base de los dedos de tus pies para dirigirte hacia la derecha. Ahora tu pie derecho será tu pie adelantado y tu pie izquierdo será tu pie atrasado (B1-2). También puedes elegir comenzar con tu pie derecho adelantado; en tal caso pivotarás de la misma manera hacia la izquierda. Siempre pivota en la dirección del pie atrasado.

Para realizar un giro sanchin, comienza en posición de guardia de sanchin con un pie adelantado sobre el otro, dedo y talón en la misma línea, y los pies separados a la anchura de los hombros. Pivota en la base de los dedos de tu pie atrasado, girando el talón hacia tu pierna de apoyo adelantada. Cuando comienzas a girar, a tu izquierda si tu pierna derecha esta adelantada, o a tu derecha si tu pierna izquierda esta adelantada, gira tus caderas y hombros en la dirección del giro antes de mover tu pierna adelantada. Coloca el talón de tu pierna pivote sobre el suelo y trae tu otra pierna alrededor rápidamente de tal manera que el dedo de ese pie esté en línea con el talón de la pierna pivote. Finalmente, tu pierna adelantada será tu pierna atrasada mientras giras $180^{\circ}$ para enfrentarte a la dirección contraria desde la que empezaste (B3-4). Una vez que has dominado estos giros, estás preparado para el ciclo de las "Cuatro Direcciones".

Comienza el ciclo de las "Cuatro Direcciones" colocándote en la posición de guardia de sanchin con tu pie derecho adelantado (C1). Da un paso hacia delante con el pie izquierdo (C2). Luego, pivota $90^{\circ}$ a tu derecha (C3). Ahora realiza un giro sanchin, girando $180^{\circ}$ hacia tu izquierda (C4).

Repite el giro pivote de $90^{\circ}$ a la derecha, seguido del giro sanchin de $180^{\circ}$ a la izquierda hasta que hayas encarado las cuatro direcciones cardinales, y estés encarando la dirección en la que comenzaste (C5-17).

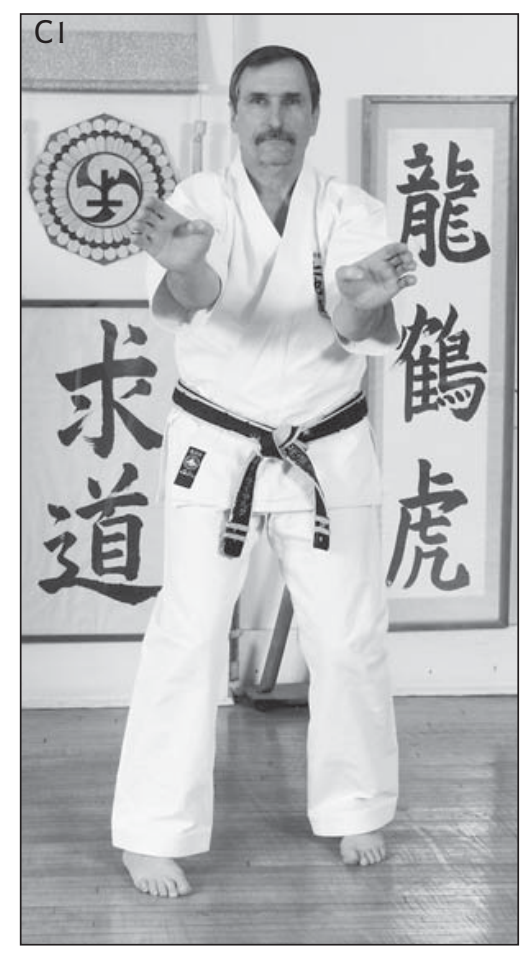




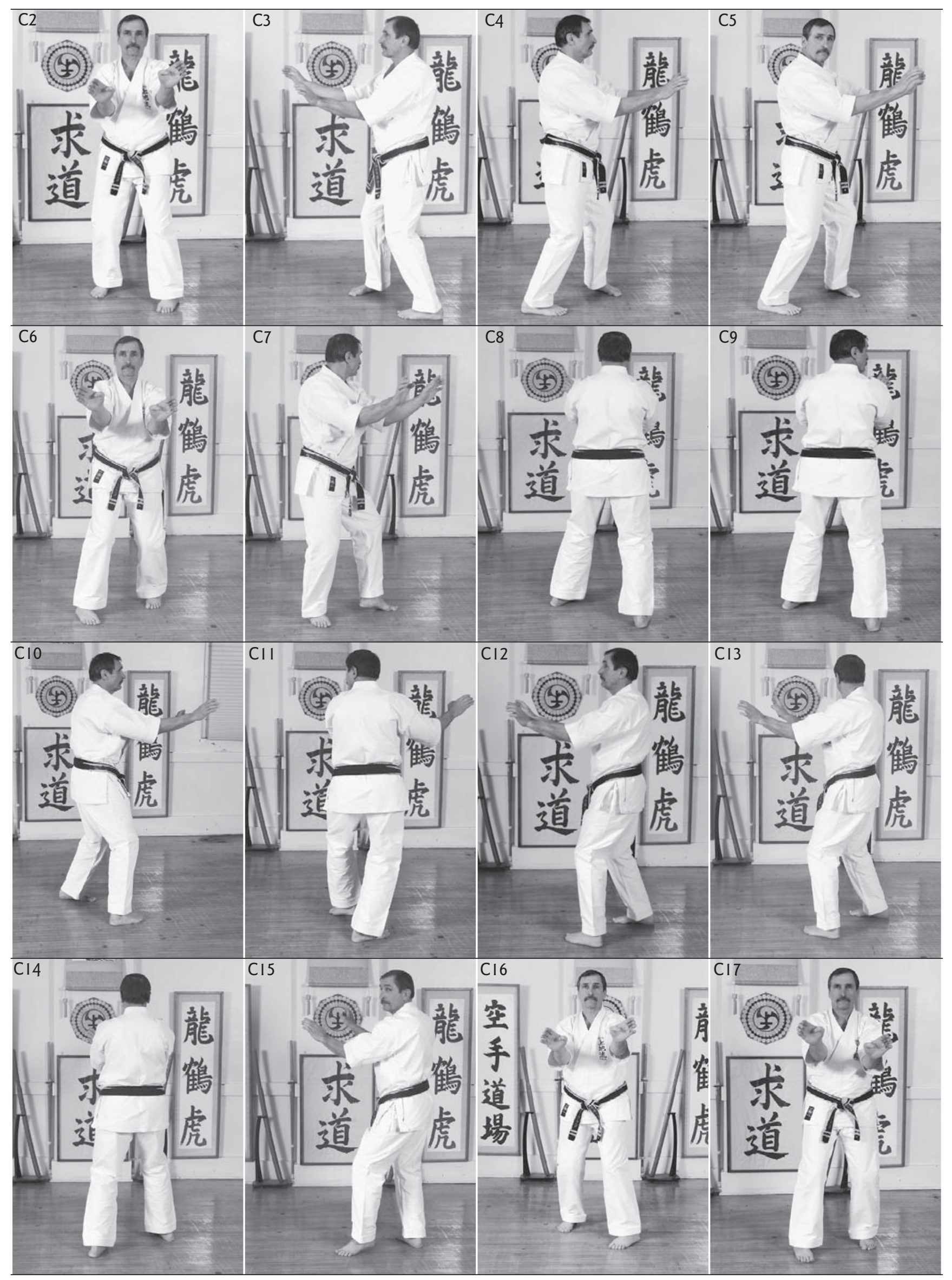

Revista de Artes Marciales Asiáticas $\diamond$ Volumen 3 Número 1 (80-91) - 2008 


\section{Práctica de las cuatro direcciones}

Una vez que has dominado este patrón de giros, estarás preparado para añadir los ejercicios de hojoundo. Para hacer esto, simplemente ejecuta un ejercicio de hojoundo después de cada giro. Puedes realizar la misma habilidad durante todo el ciclo, o puedes hacer una habilidad diferente entre cada giro. En D1-36 se muestra un ciclo completo de la técnica de las "Cuatro Direcciones" aplicando el ejercicio del bloqueo en círculo, puñetazo de gancho. Este conjunto comienza con un paso hacia delante seguido de un paso hacia atrás antes de comenzar el ciclo (D1-7).

DI-4 Desde la posición de guardia izquierda, da un paso hacia delante con tu pierna derecha. Realiza un bloqueo en círculo con la izquierda frente a la pierna atrasada y un puñetazo en gancho con la derecha frente a la pierna adelantada. Vuelve siempre a la posición de guardia después de completar cada combinación.

D5-7 Da un paso hacia atrás con tu pierna derecha y realiza un bloqueo en círculo con la derecha seguido de un puñetazo en gancho con la izquierda.
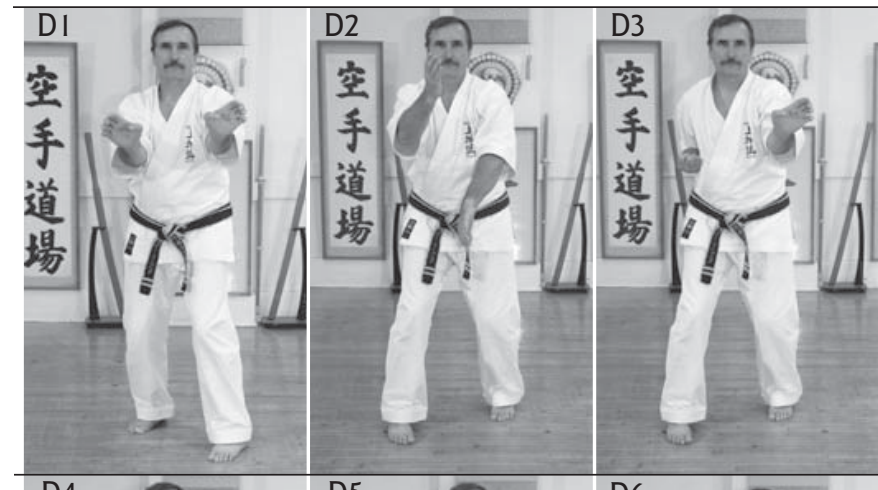

D7-10 Después de realizar el gancho con la izquierda, vuelve a la posición de guardia. Pivota $90^{\circ}$ en la base de los dedos de tus pies a la derecha, y ejecuta un bloqueo en círculo con la izquierda y un puñetazo en gancho con la derecha.

DI0-14 Desde la guardia, gira $180^{\circ}$ y ejecuta un bloqueo en círculo frente a la pierna atrasada y un puñetazo en gancho frente a la pierna adelantada. Vuelve a la posición de guardia.

D 14-17 Cambia tu peso ligeramente a las bases de los dedos de tus pies y pivota $90^{\circ}$ a la derecha. Realiza una combinación de bloqueo en círculo y puñetazo en gancho.

DI7-2I Desde la

posición de guardia, realiza el segundo giro de $180^{\circ}$. Realiza una combinación de bloqueo en círculo y puñetazo en gancho.

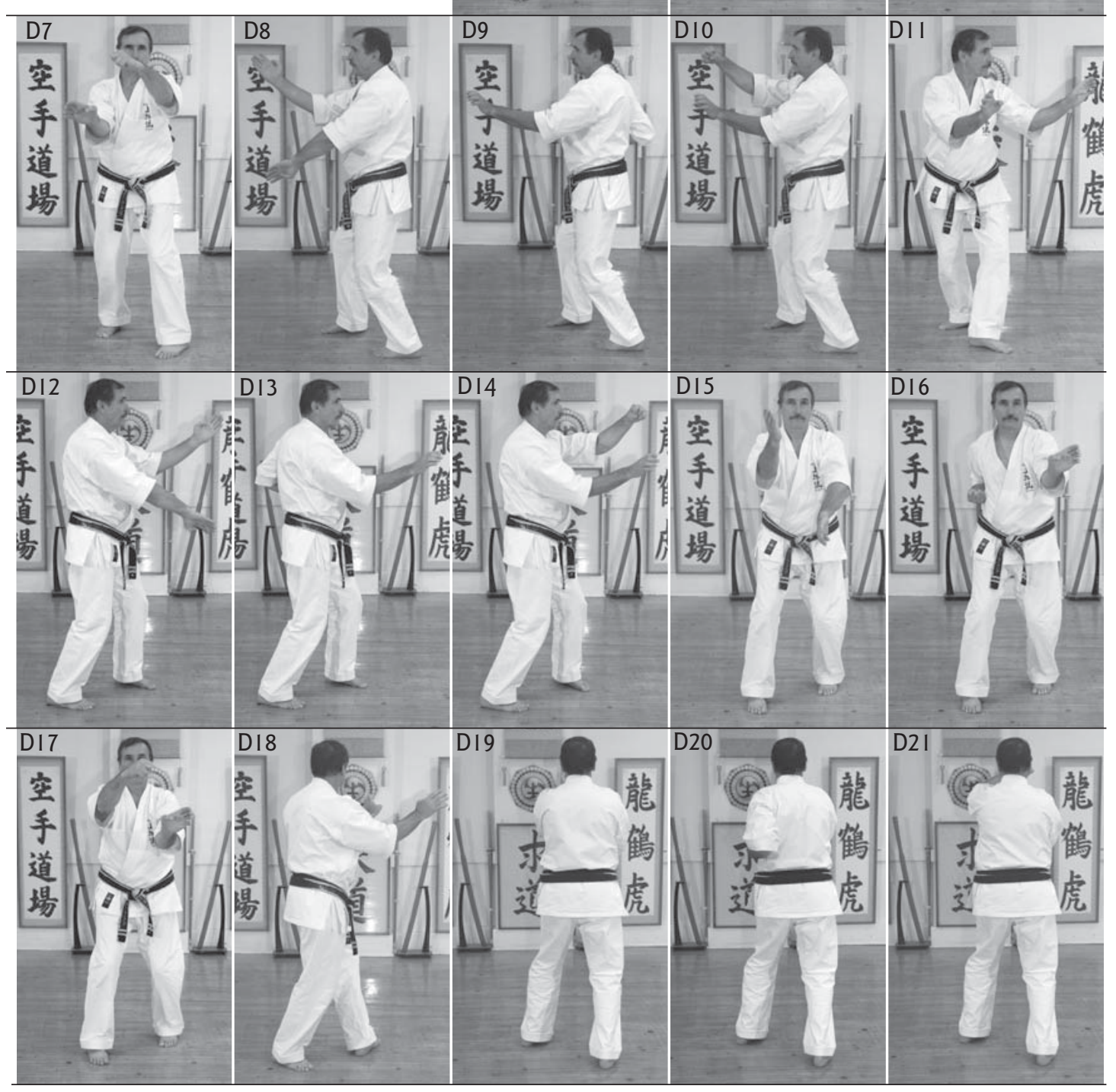




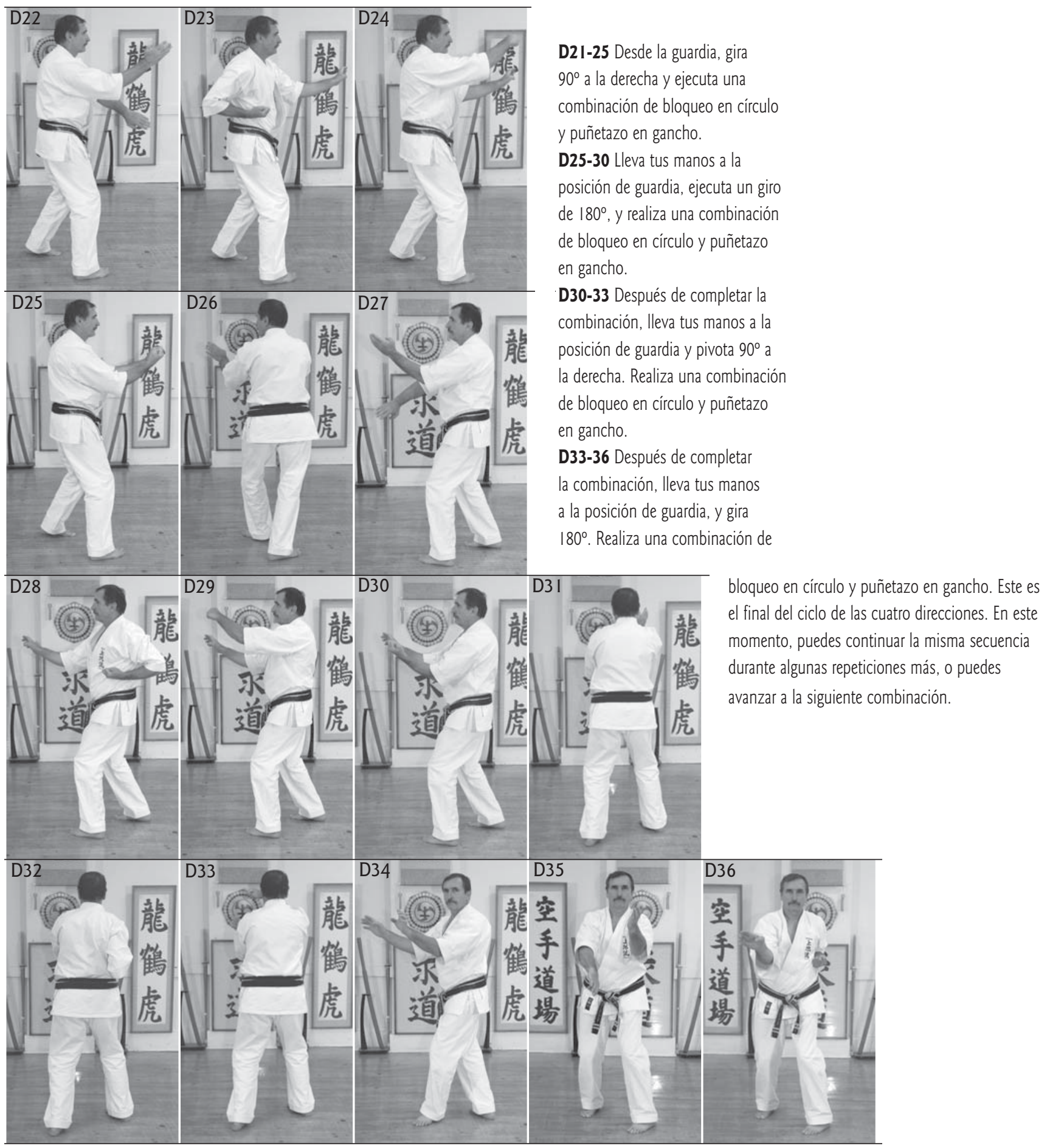

"Defendiendo en las Cuatro Direcciones" puede aplicarse a la mayoría de los ejercicios de hojoundo, excepto para la patada lateral en látigo, y las tres habilidades de avance. Recuerda que puedes practicar las "Cuatro Direcciones" empezando con cualquier pie delante, como demandan los ejercicios de hojoundo que estás deseando practicar. Si estás entrenando solo, puedes cambiar la dirección del flujo del ejercicio en cualquier momento pivotando en una dirección a tu elección. Por ejemplo, puedes desear comenzar con el pie derecho adelantado; en ese caso podrías simplemente pivotar a tu izquierda en vez de a tu derecha, y luego ejecutar el giro sanchin del modo habitual. También puedes desear añadir pasos hacia delante o hacia atrás entre los giros. Para el entrenamiento en grupo, asegúrate de establecer primero la dirección del movimiento para reducir la confusión y las posibles colisiones. 


\section{Aplicación de las cuatro} direcciones

Puedes encontrar esta técnica desafiante al principio, o encontrar que tu anteriormente sólida estructura de movimientos ahora se descoordina. Mientras continuas practicando en las cuatro direcciones, tu mente se agudizará y tu cuerpo creará una nueva memoria muscular que hará más fácil la ejecución de la técnica. Serás capaz de afinar tus habilidades, e incluso puedes encontrar que utilizas las técnicas de hojoundo más a menudo en las aplicaciones de defensa personal. Un ejemplo de cómo puede ser aplicada la técnica de "Defendiendo en las Cuatro Direcciones" se muestra en E1-25, utilizando el bloqueo en círculo y puñetazo en gancho.

E I-3 El defensor a la izquierda avanza y realiza un bloqueo en círculo para comprobar la guardia izquierda del atacante seguido de un puñetazo en gancho.

E4-6 Desde la posición de guardia, el defensor da un paso hacia atrás contra el puñetazo del atacante. Luego ejecuta un bloqueo en círculo y termina con un puñetazo en gancho.

E7-10 El defensor (a la derecha) es amenazado por un ataque. Pivota $90^{\circ}$ a la derecha y neutraliza el puñetazo con la izquierda con un bloqueo en círculo. Luego contra-ataca con un puñetazo en gancho.

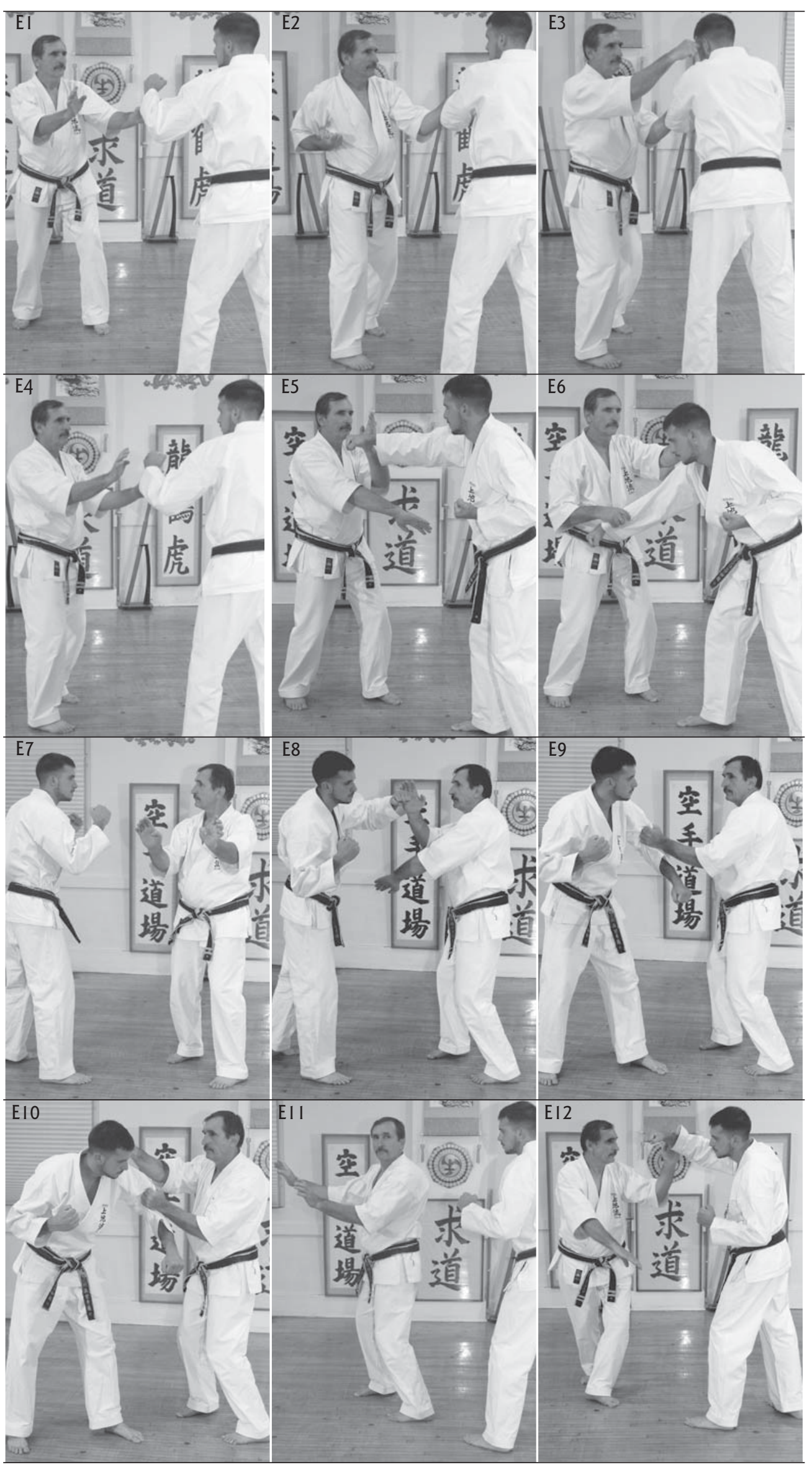

Defendiendo en las cuatro direcciones... $\diamond$ Ihor Rymaruk 


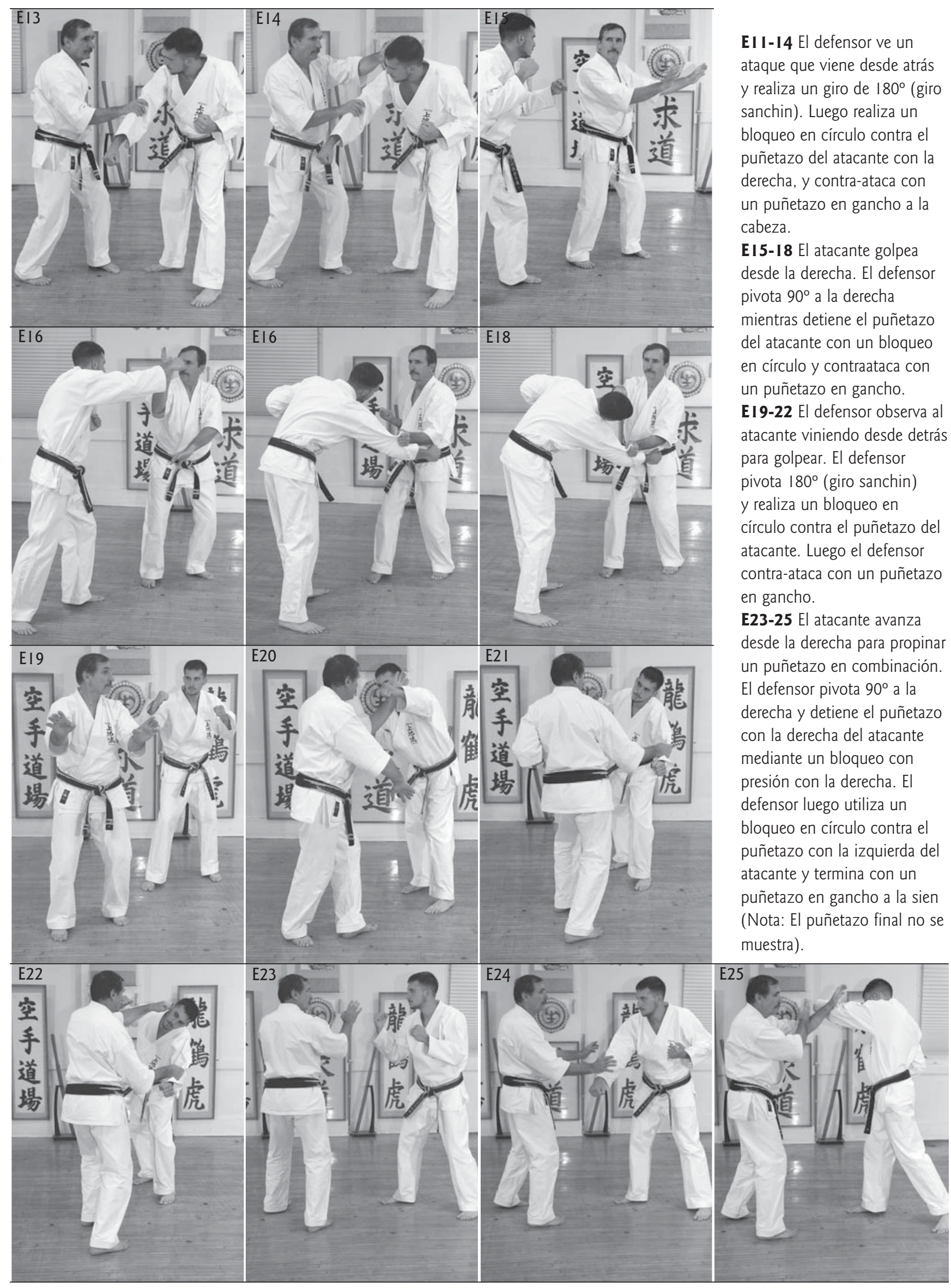

Revista de Artes Marciales Asiáticas $\diamond$ Volumen 3 Número 1 (80-91) - 2008 


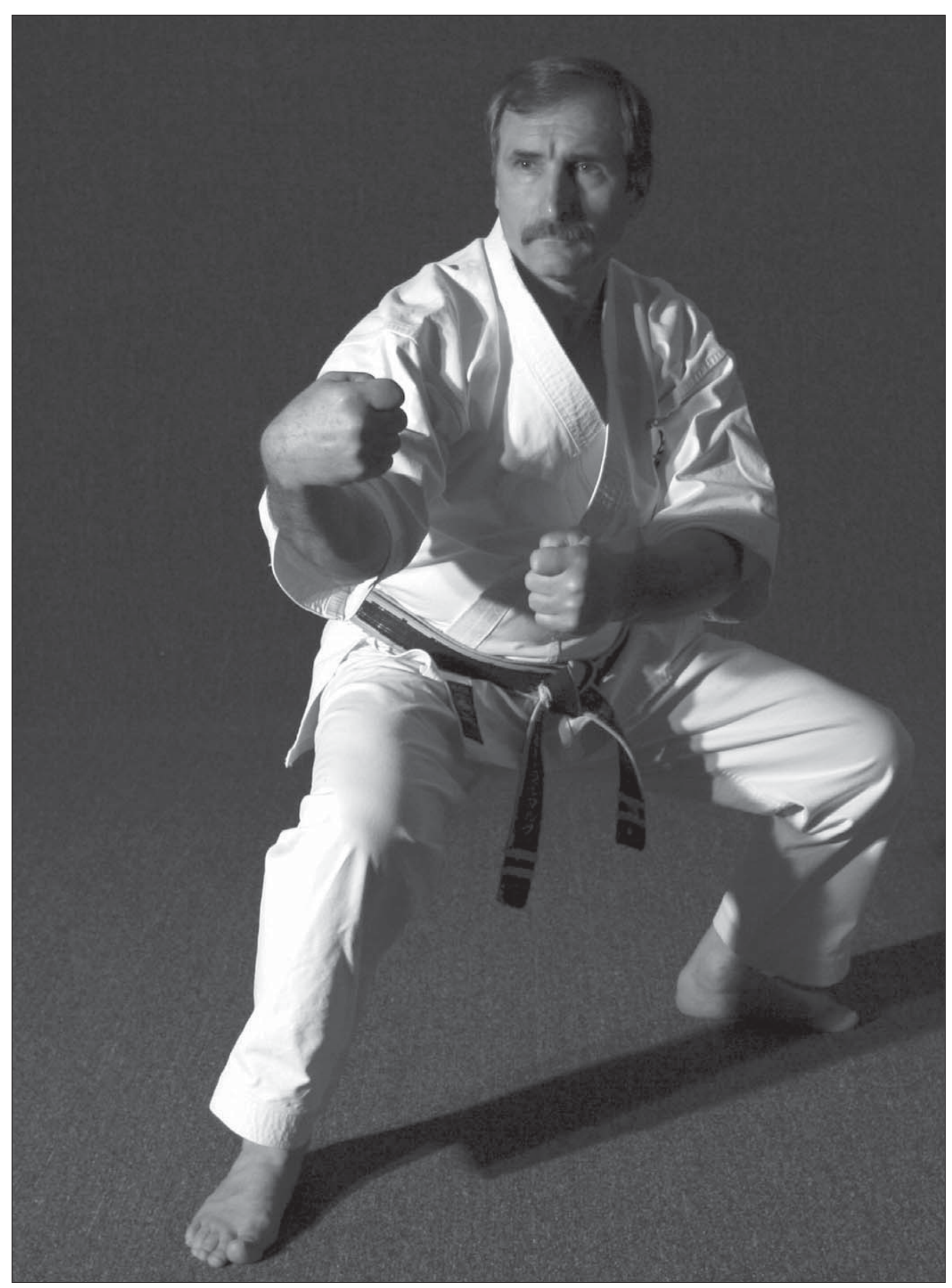




\section{Conclusión}

No suele ser habitual que uno pueda añadir un simple pero único giro a un sistema o estilo que ya ha sido probado en sí mismo y se ha enfrentado al paso del tiempo. Estoy satisfecho por haber introducido y compartido contigo mi forma especial de practicar el hojoundo para los estudiantes avanzados. A menudo, durante los años, los estudiantes avanzados se han quejado de que se aburrían con la misma rutina, o simplemente que necesitaban algo más para desafiarles. Éstos eran los menos, estudiantes que simplemente no apreciaban la efectividad de la simplicidad. La creencia era que el secreto para la aplicación efectiva de la defensa personal estaba escondido en técnicas complejas. Y hasta ahora, éstos eran los mismos estudiantes que no podían moverse en cualquier dirección sin perder cierto control de los fundamentos. Los estudiantes aprenden lo que se espera de ellos, y hoy en día tenemos que esperar más de nuestros estudiantes. Ellos no son sólo el futuro del Uechi-ryu, sino que también son los responsables de su supervivencia en un mundo muy competitivo. El entrenamiento del Uechi-ryu tiene que evolucionar para enfrentarse a las demandas y necesidades de una sociedad que esta siendo más consciente de la importancia de la auto-conservación. El kárate Uechi-ryu sobrevivirá mientras exista la necesidad de un sistema de defensa personal formidable. Un estudiante que realmente está consiguiendo dominar el Uechi-ryu sin duda alguna dará la bienvenida y manejará el desafío de elevar su entrenamiento a una nueva dimensión. Es como un jugador de dardos que siempre acierta en el centro de la diana y ahora busca un nuevo desafío poniendo la diana en movimiento. De repente el experto se convierte en principiante.

He llevado mi Uechi-ryu desde una práctica estática, a moverse hacia delante y hacia atrás, a moverse en las cuatro direcciones cardinales. Aquel primer paso que tomé hace años para convertir mi práctica de Uechi-ryu en más real ha dado lugar a los muchos pasos y cambios en direcciones que han dado lugar a una rutina de trabajo mucho más realista para los estudiantes experimentados. Mientras que los estudiantes primero tienen que aprender ciertos movimientos y a desarrollar la sincronización, la distancia, y los reflejos adecuados para afrontar una situación dada de una forma segura y constructiva, llega un momento en el que las habilidades desarrolladas tienen que ponerse en movimiento.

A menudo el hojoundo se practica como una entidad separada, relegado a ser un calentamiento para la siguiente fase de formación de las habilidades. Sólo tiene sentido trabajar las habilidades que más se han practicado, ahondar en el estilo, y continuar formándolas, dándoles una utilización más real. "Defendiendo en las Cuatro Direcciones" se construye sobre las enseñanzas tradicionales y añade nuevas dimensiones y desafíos a los practicantes avanzados del Uechi-ryu, y sin embargo su simplicidad la hace muy adaptable para ser utilizada en otras artes marciales. Se dice que "una marea alta eleva todos los barcos en el puerto". Por lo tanto, si mis simples modificaciones tienen mérito, son aceptadas y llegan a ser utilizadas abiertamente por mis compañeros maestros, entonces habré contribuido a avanzar en nuestro conocimiento de la esencia del Uechi-ryu.

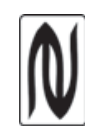

\section{AGRADECIMIENTOS ESPECIALES A:}

Jennifer Rymaruk, por la ayuda en la edición y la fotografía, Justin Rymaruk, por ser mi compañero en las demostraciones, y Amber Vosko, por la ayuda técnica. 